\title{
Ketamine and sleep modulate neural complexity dynamics in cats
}

3 Claudia Pascovich ${ }^{1,2}$, Santiago Castro-Zaballa ${ }^{1}$, Pedro A.M. Mediano ${ }^{2}$, Daniel Bor $^{2}$, Andrés

4 Canales-Johnson ${ }^{2,3}$, Pablo Torterolo ${ }^{1 *}$ and Tristan A. Bekinschtein ${ }^{2 *}$.

6 1. Laboratory of Sleep Neurobiology, Department of Physiology, School of Medicine,

7 Universidad de la República, Uruguay.

8 2. Consciousness and Cognition Laboratory, Department of Psychology, University of

9 Cambridge, $U K$

10 3. Vicerrectoría de Investigación y Posgrado, Universidad Católica del Maule, Talca, Chile

11

$12 *$ Shared senior authors

13 Correspondence: cpascovich@gmail.com (C.P.) 


\section{Abstract}

There is increasing evidence that level of consciousness can be captured by neural informational complexity: for instance, complexity, as measured by the Lempel Ziv (LZ) compression algorithm, decreases during anesthesia and non-rapid eye movement (NREM) sleep in humans and rats, when compared to LZ in awake and REM sleep. In contrast, LZ is higher in humans under the effect of psychedelics, including subanesthetic doses of ketamine. However, it is both unclear how this result would be modulated by varying ketamine doses, and whether it would extend to other species. Here we studied LZ with and without auditory stimulation during wakefulness and

24 different sleep stages in 5 cats implanted with intracranial electrodes, as well as under 25 subanesthetic doses of ketamine (5,10, and $15 \mathrm{mg} / \mathrm{kg}$ i.m.). In line with previous results, $\mathrm{LZ}$ was 26 lowest in NREM sleep, but similar in REM and wakefulness. Furthermore, we found an inverted

27 U-shaped curve following different levels of ketamine doses in a subset of electrodes, primarily in 28 prefrontal cortex. However, it is worth noting that the variability in the ketamine dose-response curve across cats and cortices was larger than that in the sleep-stage data, highlighting the differential local dynamics created by two different ways of modulating conscious state. These results replicate previous findings, both in humans and other species, demonstrating that neural complexity is highly sensitive to capture state changes between wake and sleep stages while adding

33 a local cortical description. Finally, this study describes the differential effects of ketamine doses,

34 replicating a rise in complexity for low doses, and further fall as doses approach anesthetic levels 35 in a differential manner depending on the cortex.

\section{Keywords}




\section{Introduction}

There is increasing evidence for a strong association between neural information measures, such as electrophysiological signal complexity, and level of consciousness (Abásolo et al., 2015;

50 Castro-Zaballa et al., 2019; Mateos et al., 2018; Schartner et al., 2015; Schartner, 2017; Schartner, 2017; Zhang et al., 2001). One of the most studied neural complexity metrics is Lempel-Ziv complexity (LZ), capturing the number of distinct substrings or patterns within a sequence (Lempel \& Ziv, 1976; Ziv \& Lempel, 1978). A decrease in complexity has been demonstrated for anesthesia (Li \& Mashour, 2019; Schartner et al., 2015; Zhang et al., 2001), and during non-rapid eye movement sleep (NREM sleep) when compared to normal wakefulness. However, REM complexity has consistently been shown to be above NREM sleep and below normal wakefulness (Abásolo et al., 2015; Andrillon et al., 2016; Mateos et al., 2018; Schartner et al., 2017). The increase in complexity during REM, where vivid dreaming often occurs, may lend credence to the hypothesis that complexity may not only be modulated by consciousness level but also signal the degree of contents of consciousness (Abásolo et al., 2015; Mateos et al., 2018).

Further evidence for LZ associated with an increase in the range of conscious contents comes from higher LZ during resting state in humans under the effect of psychedelics, specifically LSD, psilocybin, and subanesthetic doses of the dissociative NMDA-antagonist ketamine, compared to placebo (Li \& Mashour, 2019; Mediano et al., 2020; Schartner, et al., 2017). These drugs have profound and widespread effects on conscious experiences, both internally and externally generated. More specifically, they appear to "broaden" the scope of conscious contents, vivifying imagination and positively modulating the flexibility of cognition (Carhart-Harris et al., 2016;

69 Carhart-Harris et al., 2014). For all three drugs, reliably higher spontaneous signal diversity was reported. More recently, a higher level of complexity following a subanesthetic dose of ketamine was also reported (Li et al., 2019; Farnes et al., 2020) in spontaneous high-density scalp

72 electroencephalography (EEG) signals in healthy volunteers, but no increase was observed when 73 auditorily stimulated.

75 Ketamine also appears to maintain spatiotemporal complexity, as measured through the 
the spatiotemporal pattern of cortical activation evoked by transcranial magnetic stimulation

78 (TMS), and has proven to be a reliable classifier of level of consciousness (Casali et al., 2013).

79 PCI decreases during propofol, midazolam and xenon anesthesia (Casali et al., 2013), but

80 maintains wakefulness baseline level during ketamine anesthesia (Sarasso et al., 2015).

82 Despite this body of work, important questions remain unanswered. First, prior studies provide 83 only a disjointed picture by investigating the effect of anesthetic dose in TMS-evoked cortical 84 activation (Sarasso et al., 2015) or subanesthetic dose in spontaneous MEG signals (Schartner et 85 al., 2017). For a more complete understanding of ketamine's psychoactive effects, a systematic 86 investigation of the dose-dependent effects of ketamine on cortical complexity using the same 87 modality is required. Therefore, in this work we aimed to investigate the level of informational complexity during different stages of sleep in the cat as well as under subanesthetic doses of ketamine in a dose-dependent manner, compared to the control awake state. Additionally, we

90 determined how the complexity measures under ketamine compared to baseline conditions, with 91 or without the presence of sensory stimulation. Finally, we sought to understand the possible 92 differences in informational complexity between resting-state periods and sensory stimulation

93 periods across conscious states, in an attempt to characterize the interaction between psychedelic 94 states and perturbational states. Accordingly (Pascovich et al., 2019), the following hypotheses 95 were proposed: (1) LZ would reflect sleep level: LZ in wakefulness would be just above REM 96 sleep. REM sleep would be above light sleep (LS), and NREM sleep would have the lowest complexity value; (2) LZ would be increased during the initial period of drug infusion compared to baseline wakefulness; (3) the level of complexity would be higher under sensory stimulation

99 compared to baseline, for both conditions, with and without ketamine; and (4) stimulation-induced complexity increase would be more evident under the effect of ketamine.

\section{Results}

106 Cats underwent a polysomnographic recording in semi-restricted conditions where they were 107 adapted to sleep. Data were obtained during spontaneously occurring quiet wakefulness, LS, 
108 NREM sleep and REM sleep (Figure 1A). LZ was computed using the LZ78 algorithm (Ziv \& 109 Lempel 1978; Figure 1C) from the different sleep stages for all the cortices available (Figure 2A).

110 Effect sizes for differences between states at the single subject level are shown in Figure 2B. For 111 all animals, LZ scored higher for wakefulness than NREM sleep (Cohen's $\mathrm{d}>0.8$ ) for most of the 112 cortices. As predicted, LZ values were highest for REM and W, intermediate for LS, and lowest 113 for NREM.

115 Additionally, mixed effects models were formulated for each cortex including the cat as a random 116 effect when applicable. Thereafter, model selection was performed between linear and quadratic 117 models using Bayes Factors (BF) to decide between U-shaped and linear fits. All model 118 comparisons between linear and non-linear quadratic fits showed the supremacy of the non-linear 119 fit (Table 1) in agreement with our previous hypotheses, where the REM sleep showed higher 120 complexity values than the deep sleep - with the exception of the right somatosensory cortex, 121 where the results showed a flattening of the curve compared to all other cortices (Figure 2).

A

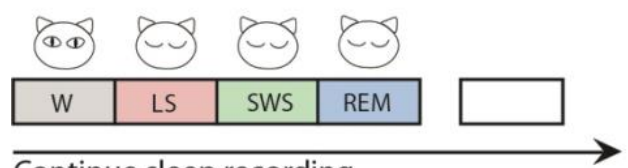

Continue sleep recording

B

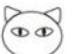

Ketamine effect

(D) with stimulation

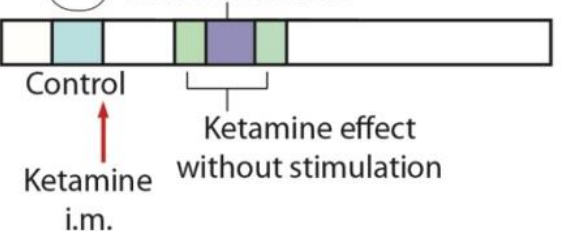

C

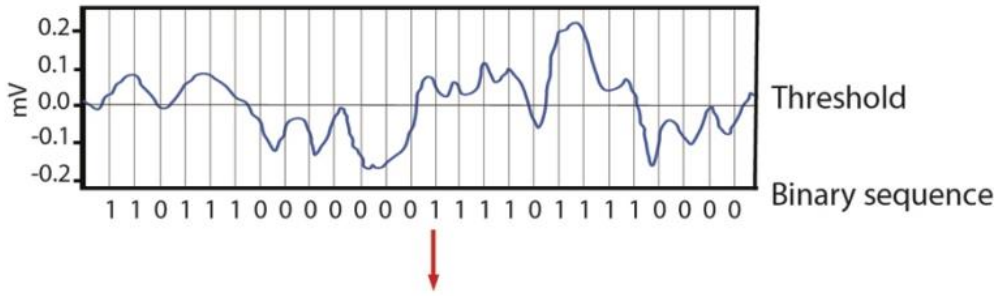

$1|10| 11|100| 0|00| 001|111| 01 \mid 11100000$ Complexity

Analysis

Result

124 FIGURE 1. Schematic illustrating the experimental design for electrocorticographic recordings

125 during the different states of sleep (A) and before, and after the different doses of ketamine (B).

126 i.m., intramuscular. (C) Illustration showing how to transform a segment of EEG signal series into

127 a binary sequence and the result of the LZ complexity analysis on the binary sequence. 
A
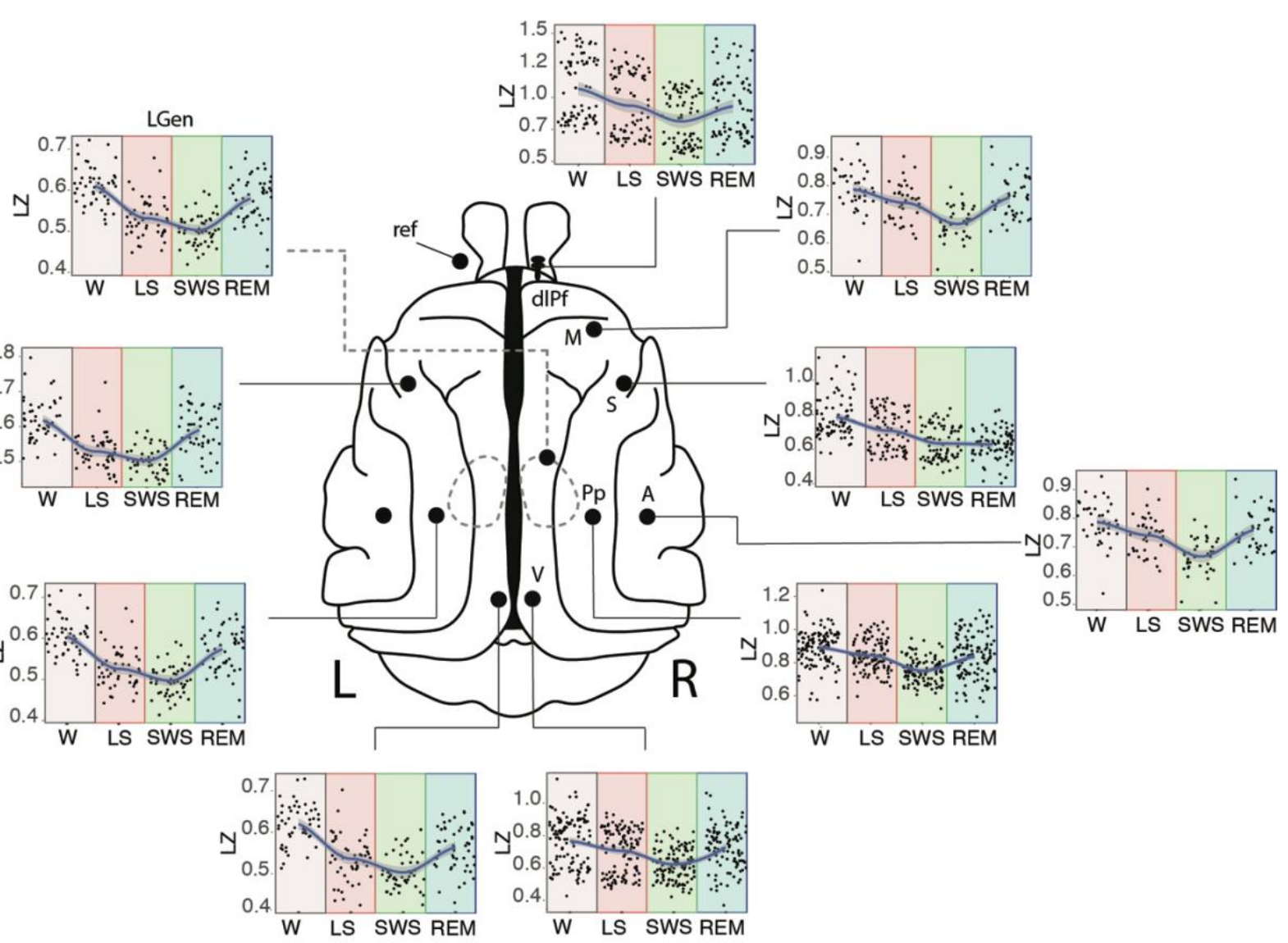

B

CAT 1

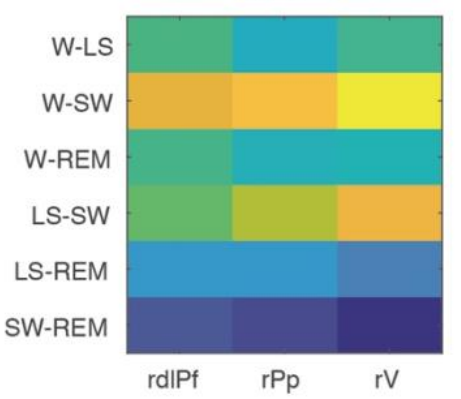

CAT 4

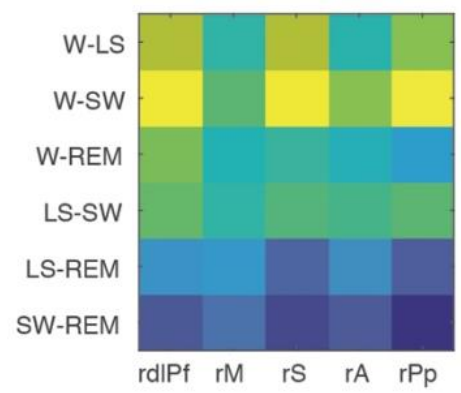

CAT 2

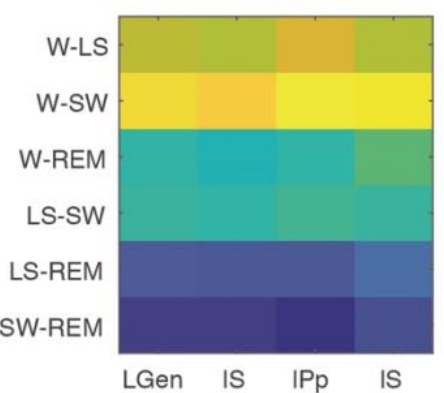

\section{CAT 5}

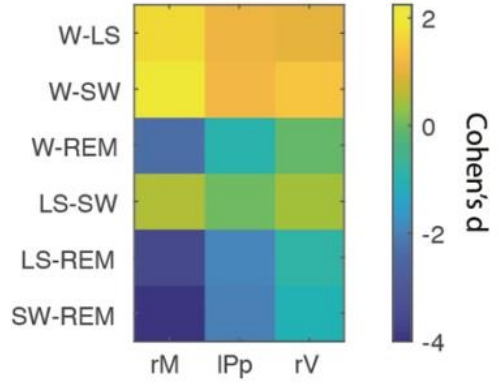

CAT 3

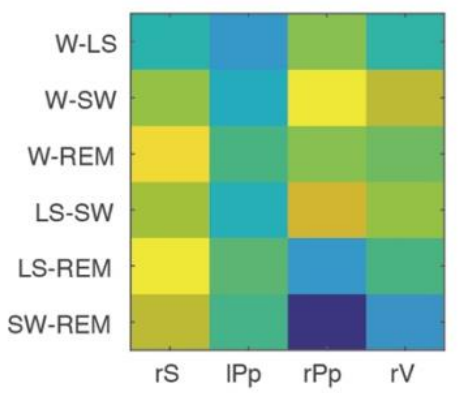

$\mathrm{dIPf}=$ Dorsolateral prefrontal cortex $\mathrm{S}=$ Primary somatosensory cortex $\mathrm{M}=$ Primary motor cortex

$A=$ Primary auditory cortex

$\mathrm{Pp}=$ Posterior parietal cortex

$\mathrm{V}=$ Primary visual cortex

LGen $=$ Lateral geniculate nucleus 
129 FIGURE 2. Cortical dynamic of $\mathbf{L Z}$ during sleep. Schematic representations of the cat brain are

130 used to visualize the differential dynamics of LZ during wakefulness and different states of sleep

131 (A), showing a U-shaped complexity curve with state progression from W to LS, SWS and REM.

132 "L" indicates Left side and "R" right side. (B) The differences in average LZ between sleep states,

133 as measured by ANOVA and Tukey post-hoc test. Effect sizes were calculated by Cohen's d and

134 represented in a colour scale, where yellow means a positive difference and blue means a negative

135 difference between the effect sizes of the pair of states compared. $\mathrm{W}=$ wakefulness; LS = light

136 sleep; SWS = slow wave sleep; REM = rapid eyes movements sleep; dlPf = dorsolateral prefrontal

137 cortex; $\mathrm{Pp}=$ posterior parietal cortex; $\mathrm{V}=$ visual cortex; LGen = lateral geniculate nucleus; $\mathrm{S}=$

138 somatosensory cortex; $\mathrm{M}$ = motor cortex; $\mathrm{A}$ = auditory cortex; ref, reference electrode location. In

139 Figure B, "r" indicates right and "l" indicates left cortex.

140

141

\begin{tabular}{|c|c|c|c|}
\hline Cortex & № of cats & Model & BF \\
\hline \multirow[t]{2}{*}{ Right dorsolateral prefrontal } & 2 & linear & \\
\hline & & quadratic * & $6.88 \times 10^{18}$ \\
\hline \multirow[t]{2}{*}{ Right primary motor } & 2 & linear & \\
\hline & & quadratic * & $1.90 \times 10^{17}$ \\
\hline \multirow[t]{2}{*}{ Right primary auditory } & 1 & linear & \\
\hline & & quadratic * & $4.42 \times 10^{6}$ \\
\hline \multirow[t]{2}{*}{ Right primary somatosensory } & 2 & linear & \\
\hline & & quadratic & 0.681 \\
\hline \multirow[t]{2}{*}{ Left primary somatosensory } & 1 & linear & \\
\hline & & quadratic * & $8.53 \times 10^{20}$ \\
\hline \multirow[t]{2}{*}{ Right posterior parietal } & 3 & linear & \\
\hline & & quadratic * & $1.76 \times 10^{7}$ \\
\hline \multirow[t]{2}{*}{ Left posterior parietal } & 1 & linear & \\
\hline & & quadratic * & $7.19 \times 10^{9}$ \\
\hline \multirow[t]{2}{*}{ Right primary visual } & 3 & linear & \\
\hline & & quadratic * & $3.70 \times 10^{11}$ \\
\hline \multirow[t]{2}{*}{ Left primary visual } & 1 & linear & \\
\hline & & quadratic * & $3.77 \times 10^{17}$ \\
\hline \multirow[t]{2}{*}{ Right Lateral Geniculate } & 1 & linear & \\
\hline & & quadratic * & $7.00 \times 10^{20}$ \\
\hline
\end{tabular}

142 TABLE 1. Selection between linear and non-linear models among different sleep stages for

143 each cortex. Mixed effects models were formulated for each cortex including the cat as a random 
144 effect when applicable. Bayes Factors (BF) were used to decide between U-shaped and linear fits.

145 With the exception of right primary somatosensory cortex, all model comparisons showed the

146 supremacy of the quadratic fit. The asterisks indicate substantial evidence for a quadratic fit (BF

$147>5)$.

\section{Heterogenous cortical dynamics across cortices under ketamine}

152 For this experiment, the data had been collected under the same experimental conditions than for

153 sleep recordings in the same cats, and i.m. injections of ketamine of 5,10 or $15 \mathrm{mg} / \mathrm{Kg}$ were 154 performed in separate non-consecutive days as schematized in Figure 1B (see Methods). Again, 155 LZ was calculated in epochs before and after the administration of the drug. To address dose156 response relationships, a multilevel model was used where LZ was predicted by dose (fixed effect),

157 and cat and session were considered as random effects (with sessions nested within cats, and each 158 dose of ketamine was repeated four times).

160 Considerably greater LZ variability was observed under ketamine than for the sleep results, 161 especially during the lowest doses explored (Figure 3A). In some regions, the results were in 162 agreement with our hypothesis, which predicted an increase in informational complexity after the 163 lowest ketamine dose, followed by a decrease after the higher dose showing an inverted U-shaped 164 relationship. This was observed clearly in right rostral and dorsolateral prefrontal cortices as well 165 as the right primary auditory cortex $\left(\mathrm{BF}=1.70 \times 10^{14}, \mathrm{BF}=2.57 \times 10^{5}, \mathrm{BF}=8.25 \times 10^{9}\right.$, respectively). 166 However, when we look at the individual effect per cortex in each animal, it can be seen that the 167 inverted U-shaped relationship is not systematic between cats and is present only in the cortices of 1682 out of 5 cats (Figure $3 \mathrm{~B})$.

170 On the other hand, an opposite curve was obtained for somatosensory and posterior parietal 171 cortices. Finally, for the visual cortex the effects were less consistent among cats; in this last 172 example, the two cats tested had different responses to ketamine with opposite effects (Figure 3B). 
173 As for sleep, we studied ketamine effects on LZ using model fitting of the individual mixed effects

174 models for each cortex. Model selection was performed in this case between linear, quadratic and 175 cubic models using BF (Table 2).

A
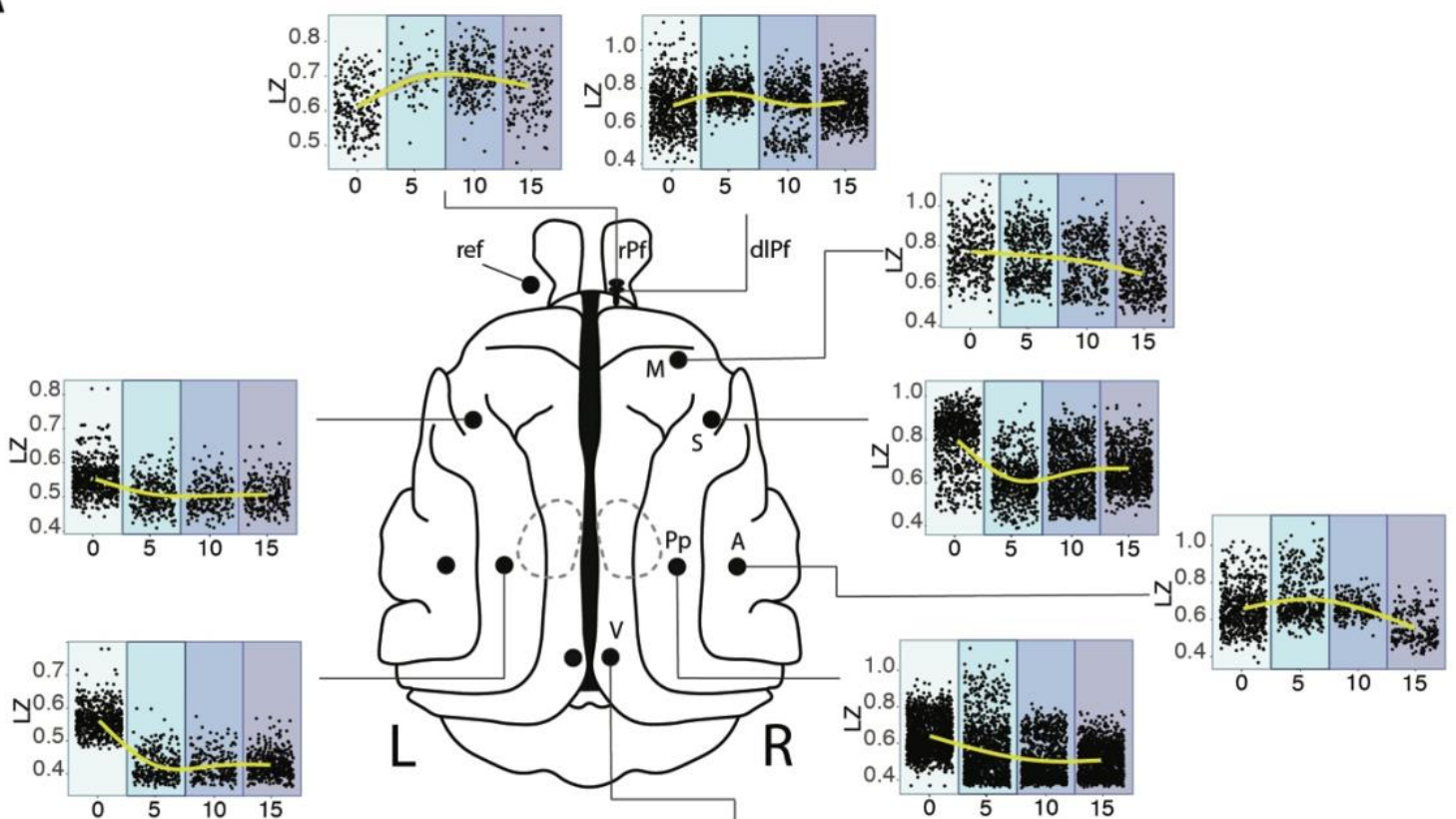

B
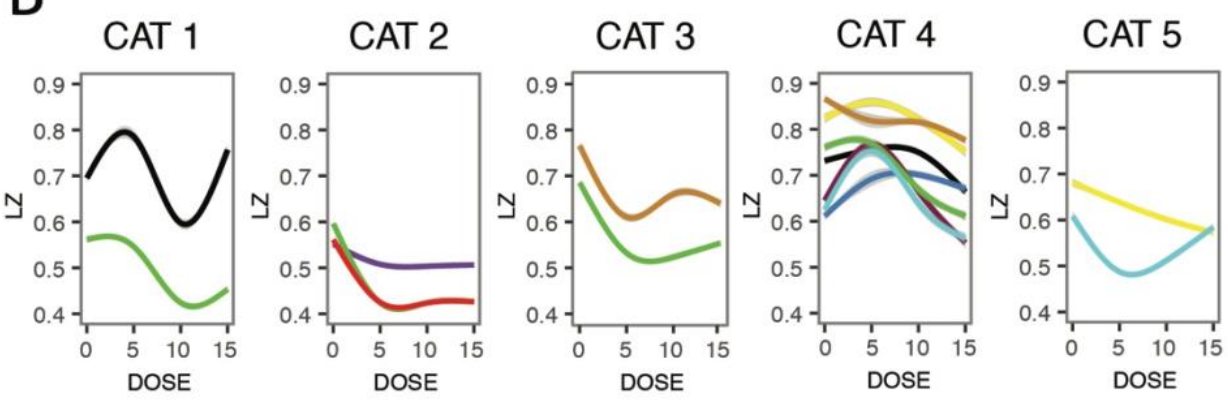

- Right rostal prefrontal cortex

- Right dorsolateral prefrontal cortex

10 Right primary motor cortex

- Right primary somatosensory cortex

- Left primary somatosensory cortex

- Right primary auditory cortex

- Right posterior parietal cortex

- Left posterior parietal cortex

night primary visual cortex

FIGURE 3. Curves dose-response of the dose of ketamine on cortical dynamic of LZ. (A) Dose-response curve of subanesthetic doses of ketamine, showing an inverted U-shaped curve only for prefrontal and auditory cortices, with monotonic decrease of complexity with

182 concentration for the other cortices. Each plot represents the sum of the different sessions for each

183 dose of the different cats which have that cortex, therefore the $\mathrm{N}^{\circ}$ of cats is different per cortex. 
184 (B) The curves are plotted per cat. It can be clearly seen that the variability in the informational 185 complexity dynamic per cortex and per cat is evidenced more clearly when plotted individually, 186 and shows that there is a dissociation of the dose-response and the anatomical location. The doses 187 are represented in $\mathrm{mg} / \mathrm{Kg}$. W, wakefulness; LS, light sleep; SWS, slow wave sleep; REM, rapid 188 eyes movement sleep; rPf, rostral prefrontal cortex; dlPf, dorsolateral prefrontal cortex; M, primary 189 motor cortex; S, primary somatosensory cortex; A, primary auditory cortex, Pp, posterior parietal 190 cortex; V, visual cortex. "L" indicates Left side and "R" right side.

\begin{tabular}{|c|c|c|c|}
\hline Cortex & № of cats & Model & BF \\
\hline \multirow[t]{3}{*}{ Right dorsolateral prefrontal } & 2 & linear & \\
\hline & & quadratic * & $2.57 \times 10^{5}$ \\
\hline & & cubic & 288.73 \\
\hline \multirow[t]{2}{*}{ Right rostral prefrontal } & 1 & linear & \\
\hline & & quadratic * & $1.70 \times 10^{14}$ \\
\hline \multirow[t]{2}{*}{ Right primary motor } & 2 & linear & \\
\hline & & quadratic & 0.04 \\
\hline \multirow[t]{2}{*}{ Right primary auditory } & 2 & linear & \\
\hline & & quadratic * & $8.25 \times 10^{9}$ \\
\hline \multirow[t]{3}{*}{ Right primary somatosensory } & 3 & linear & \\
\hline & & quadratic & $3.0 \times 10^{-3}$ \\
\hline & & cubic & 0.66 \\
\hline \multirow[t]{2}{*}{ Left primary somatosensory } & 1 & linear & \\
\hline & & quadratic & 1.08 \\
\hline \multirow[t]{2}{*}{ Right posterior parietal } & 4 & linear & \\
\hline & & quadratic & $1.13 \times 10^{-4}$ \\
\hline \multirow[t]{2}{*}{ Left posterior parietal } & 2 & linear & \\
\hline & & quadratic * & 46057.12 \\
\hline \multirow[t]{3}{*}{ Right primary visual } & 2 & linear & \\
\hline & & quadratic & 1.28 \\
\hline & & cubic & $3.0 \times 10^{-3}$ \\
\hline
\end{tabular}

194 TABLE 2. Selection between linear and non-linear models among different doses of ketamine

195 for each cortex. Mixed effects models were formulated for each cortex including the cat as a 196 random effect when applicable. Bayes Factors (BF) were used to decide between quadratic (U197 shaped), cubic and linear fits. A clear evidence towards a quadratic fit was found for right 
dorsolateral and rostral prefrontal cortices, right primary auditory cortex and left posterior parietal cortex. The asterisks indicates substantial evidence for a quadratic fit (BF $>5)$.

\section{Informational complexity is not modulated by auditory stimuli under ketamine}

In 3 cats, modulation by auditory stimuli was studied. Under control conditions without ketamine, an increase in LZ was observed during stimulation in dorsolateral prefrontal $(0.66 \pm 0.04$ to $\left.0.70 \pm 0.007, \mathrm{p}<0.01, \eta^{2}=0.044, \mathrm{BF}=10619.07\right)$ and auditory $(0.63 \pm 0.05$ to $0.66 \pm 0.007, \mathrm{p}<$ $\left.0.01, \eta^{2}=0.035, \mathrm{BF}=75.27\right)$ cortices, whereas the effect on other cortices studied were nonreliable, including right posterior parietal cortex $\left(0.53 \pm 0.02\right.$ to $0.53 \pm 0.001, \mathrm{p}<0.01, \eta^{2}=0.008$, $\left.\mathrm{BF}=7.0 \times 10^{-5}\right)$, right somatosensory cortex $\left(0.62 \pm 0.05\right.$ vs $0.62 \pm 0.002$ with $\mathrm{p}=0.61, \eta^{2}=0.005$, $\left.\mathrm{BF}=1.0 \times 10^{-4}\right)$, left somatosensory cortex $\left(0.52 \pm 0.005\right.$ vs $0.53 \pm 0.002$ with $\mathrm{p}<0.01, \eta^{2}=0.002$, $\mathrm{BF}=0.09)$, and left posterior parietal cortex $\left(0.47 \pm 0.01\right.$ vs $0.48 \pm 0.001, \mathrm{p}=0.85, \eta^{2}=0.001, \mathrm{BF}$

$211=1.0 \times 10^{-4}$, Figure 4A).

Initially we hypothesized that the increment in complexity under the sensory stimulation versus non-stimulation conditions would be more evident under the effect of ketamine. However, there was no interaction between stimulation and ketamine. For left somatosensory cortex, non-reliable effect was observed during basal conditions in response to the stimuli ( $\left.\mathrm{p}=3 \times 10^{-4}, \mathrm{BF}=0.09\right)$, and this effect remained unchanged under ketamine, and non-reliable interaction between dose and stimuli was observed $\left(\mathrm{p}=0.16 ; \mathrm{BF}=3.5 \times 10^{-4}\right.$, Figure 4B). For right somatosensory cortex, where non-reliable increase was evidenced in control conditions (Figure 4A), no reliable interaction was 220 found during ketamine effect $\left(\mathrm{p}=2.0 \times 10^{-3} ; \mathrm{BF}=0.012\right)$ with no response to the stimuli $(\mathrm{p}=0.64$; $221 \mathrm{BF}=1.5 \times 10^{-4}$; Figure $4 \mathrm{C}$ ). For the prefrontal cortex, where an increase was observed in control 222 conditions, the same effect was found under ketamine $\left(\mathrm{p}=9.4 \times 10^{-8} ; \mathrm{BF}=335.92\right)$, with non223 reliable interaction between stimuli and ketamine $\left(\mathrm{p}=0.51 ; \mathrm{BF}=2.5 \times 10^{-8}\right.$; Fig. 4D). For left 224 posterior parietal cortex, non-reliable effect was found under baseline conditions, there was no 225 effect of stimulation under ketamine $\left(\mathrm{p}=0.85 ; \mathrm{BF}=1.2 \times 10^{-4}\right)$, and the interaction also remained 226 unchanged under ketamine $\left(\mathrm{p}=0.74 ; \mathrm{BF}=2.5 \times 10^{-6}\right.$; Figure $\left.4 \mathrm{E}\right)$. Finally, for right posterior parietal 227 cortex, no effect was found under basal conditions, there was no change with the stimuli under 
228 ketamine $\left(\mathrm{p}=0.46 ; \mathrm{BF}=7.4 \times 10^{-5}\right)$, and the modulation by stimulation was non-reliable $\left(\mathrm{p}=8 \times 10^{-}\right.$

$229{ }^{3} ; \mathrm{BF}=8 \times 10^{-3}$; Figure 4F).

230

231

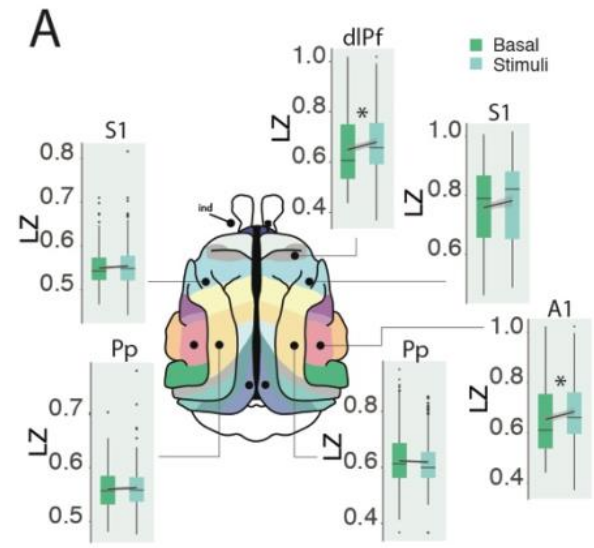

D

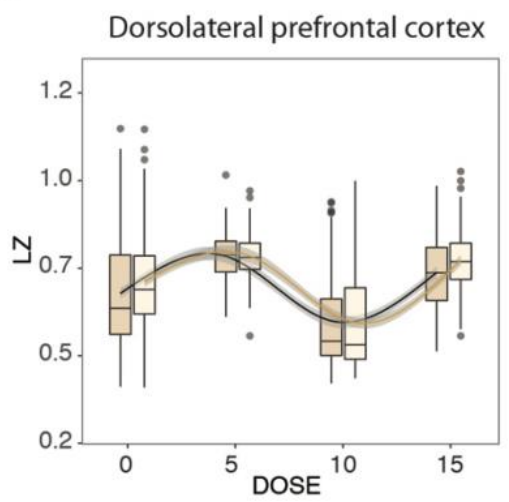

B

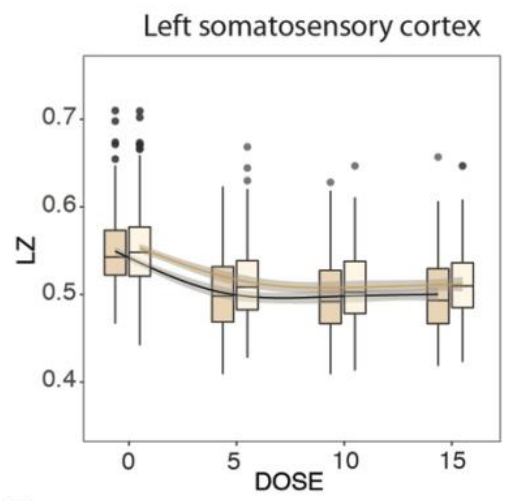

E

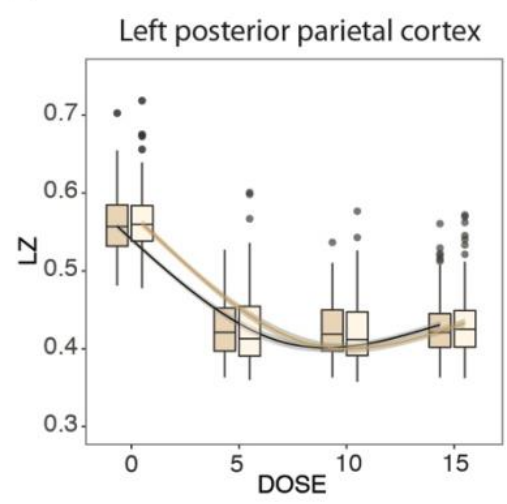

C

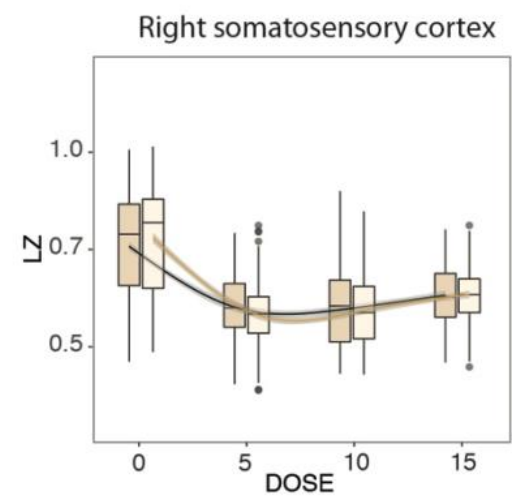

F

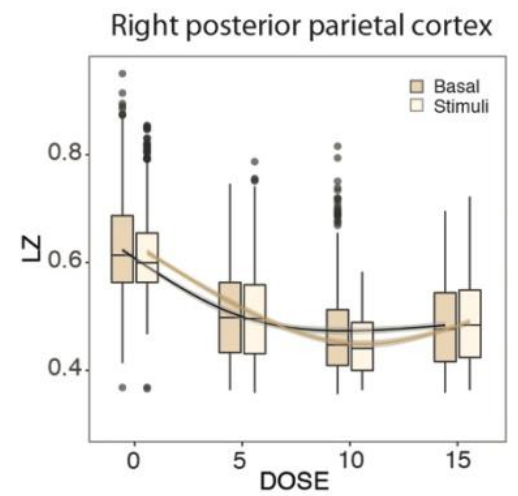

FIGURE 4. Modulation of LZ by auditory stimulation. (A) The effect of stimulation was shown without ketamine where an increase in LZ was observed during stimulation in dorsolateral prefrontal and auditory cortices. * Statistically reliable $(\mathrm{p}<0.01 ; \mathrm{BF}>5)$. dlPf $=$ dorsolateral prefrontal cortex; $\mathrm{Pp}=$ posterior parietal cortex; $\mathrm{S} 1=$ primary somatosensory cortex; $\mathrm{A} 1=$ primary auditory cortex. (B-F) Modulation by stimulation under the effect of ketamine in 5, 10 and 15 $\mathrm{mg} / \mathrm{Kg}$ doses. No stimulation by dose interaction was observed. The doses are represented in $\mathrm{mg} / \mathrm{Kg}$. 


\section{Discussion}

In this work, using LZ as a measure of dynamical complexity on direct intracranial recordings, we studied the effect of subanesthetic doses of ketamine in a dose-dependent manner. Ketamine

248 elicited a diverse set of dynamics, with the lower doses showing the most variable effects. For prefrontal and auditory cortices an increase in LZ was observed from low to medium ketamine dose. However, a decrease was evidenced at the maximum dose, drawing an inverted U-shape dose-effect curve, whereas the opposite effect was observed for other cortices including somatosensory and posterior parietal cortices, where an initial decrease was followed by an increase in complexity at higher doses. Additionally, we also presented auditory stimulation to the cats, which elicited an increase in LZ in prefrontal and auditory cortices, but this effect was not modulated by ketamine. Finally, in the same animals, we studied LZ during sleep, which by contrast show an homogeneous pattern among cortices. We demonstrate that informational complexity in the cortex and thalamus of the cat decreases in light and deep sleep compared to awake states and REM. For most of the cortex, there is only marginal complexity difference between wakefulness and REM sleep. The results were consistent among cats and similar for all the cortices studied and, more importantly, confirm previous results in humans and rats.

262 As measures of neural signal diversity are known to be sensitive to conscious level in natural state changes (the sleep-wake cycle), they are also sensitive to the changes in brain dynamics associated with psychedelic and anesthetic states. Specifically, Schartner et al. found increased global neural signal diversity for the psychedelic state induced by ketamine, psilocybin and LSD, as compared to placebo, across a range of measures (Schartner et al., 2017). Other recent MEG and EEG studies

267 have also demonstrated elevated signal diversity induced by canonical serotonergic psychedelics and ketamine (Tagliazucchi et al., 2014; Timmermann et al., 2019).

270 From the perspective of its effects on EEG signal diversity, the dissociative NMDA-antagonist

271 ketamine diverges from traditional anesthetics at subanesthetic concentrations, as it induces 272 dissociative states characterized by a maintained or enhanced repertoire of brain states (Li \& 273 Mashour, 2019; Schartner et al., 2017). This is in contrast to GABAergic anesthetics such as 274 propofol, which have been shown to degrade sensory integration and attenuate neural signal 
diversity in a dose-dependent manner (Ferenets et al., 2006, 2007; Ishizawa et al., 2016). While those studies were based on EEG signals that had been low-pass filtered at $55 \mathrm{~Hz}$ and lacked cortical dynamics in higher gamma frequencies, Pal et al. have recently demonstrated that this part of the signal is important. Using intracranial EEG data from frontal and parietal cortices of rats receiving ketamine or propofol anesthesia, they demonstrated a reduction in broadband (0.5-175 $\mathrm{Hz}$ ) EEG complexity during ketamine anesthesia that is comparable to that induced by the GABAergic anesthetic propofol. Bandwidth-specific analyses restricted to higher gamma

282 frequencies showed that ketamine anesthesia is distinguished from propofol by suppression of EEG complexity in high gamma frequencies in the range of $65-175 \mathrm{~Hz}$, which previous human studies using scalp EEG could not reveal (Pal et al., 2020). In the present study, by using intracranial electrodes in cats, we were able to study broadband $(>0.5 \mathrm{~Hz})$ signal complexity .

Contrary to the apparent convergence of psychedelics (LSD, DMT, psylocibin) reported (Schartner et al., 2017), some of us (González et al., 2021) have shown that the effects of ibogaine, a psychedelic alkaloid, induces high gamma power but are less coherent and less complex than control condition, and similar to natural REM sleep. Although some differences in the complexity measure or animal model may explain the difference, it is key to highlight that the ibogaine local complexity patterns were more consistent than those found in the current study, pointing to a different mode of action between alkaloid, serotoninergic and NMDA psychedelics.

Ketamine's primary mechanism of action is as an NMDA antagonist whose receptors are located quite ubiquitously across the cerebral cortex, as well as subcortically (Conti et al., 1994; Huntley et al., 1994). A differential interaction with various subtypes of NMDA receptors could explain the heterogeneity in cortical response under the effects of ketamine (Zanos et al., 2018). However, the non-NMDA receptor effects of ketamine cannot be discounted, in particular its interactions with opioid receptors and HCN channels (Chen et al., 2009; Zanos et al., 2018; Zhou et al., 2013). Additionally, ketamine may indirectly exert effects through its interaction with other circuits. Previous work reported that subanesthetic doses of ketamine increased the release of not only 5HT (Amargós-Bosch et al., 2006; López-Gil et al., 2012, 2019), but also noradrenaline (Lorrain et al., 2003) as well as glutamate (Moghaddam et al., 1997) in the medial prefrontal cortex, which may increase signal complexity. At the receptor level, ketamine blocks excitatory NMDA 
receptors on fast-spiking cortical interneurons more effectively than those on pyramidal neurons. This results in down-regulation of interneuron activity, and decreased GABA release at the interneuron-pyramidal neuron synapse (Homayoun \& Moghaddam, 2007; Seamans, 2008). This decrease in inhibitory tone (decreased GABA release) results in markedly excited pyramidal neurons. It has been proposed that this may explain why ketamine is associated with increased cerebral glucose utilization and blood flow (Langsjo et al., 2005; Långsjö et al., 2004), and increased EEG gamma oscillations (Blain-Moraes et al., 2014; Castro-Zaballa et al., 2019; FerrerAllado et al., 1973; Lee et al., 2013; Schwartz et al., 1974) and may also help us understand the changes observed in the complexity of the signal. However, our results show a decrease of LZ in somatosensory and posterior parietal cortices after the lowest dose of ketamine (Figure 3B). As

316 both of these cortices process somatosensory information, our results may be due to a reduction in 317 the somatosensory information influx, as one of the main effects of subanesthetic doses of 318 ketamine is analgesia (Zanos et al., 2018).

Neural diversity, assessed by LZ, is an attractive measure because of simplicity, practical applicability, and consistency with both complexity-based (Tononi et al., 2016; Tononi \& Edelman, 1998) and entropy-based (Carhart-Harris, 2018; Carhart-Harris et al., 2014) theories of neural integration and consciousness. The measure is also useful in questions regarding local processing as it is computed at the electrode level, thus was able to demonstrate differential effects in distinct thalamic and cortical brain regions. Indeed, according to the dynamic core hypothesis

326 (Tononi \& Edelman, 1998) and subsequent theoretical developments such as Information

327 Integration Theory (Tononi et al., 2016), only certain distributed subsets of the neuronal groups 328 that are activated or deactivated in response to a given task are associated with conscious 329 experience, therefore a large cluster of neuronal groups that together constitute, on a time scale of 330 hundreds of milliseconds, a unified neural process of high complexity can be termed the "dynamic 331 core". In line with this idea, our results could be interpreted as the prefrontal and auditory cortices, 332 where an increase in LZ was observed under the 5mg dose of ketamine, constituting a part of the 333 “dynamic core", and somatosensory and posterior parietal cortices playing a different role in neural 334 integration. 
Another useful framework for understanding these results is the neuroscience of arousal, including wakefulness, sleep, circadian rhythms, responsiveness and alertness (Bekinschtein et al., 2009; Brown et al., 2011). Sleep shows a clear change in arousal throughout the day cycle; the intensity of the stimuli needed to wake up a person is maximal in deep sleep and lower in light sleep and REM. This pattern partially mimics the results obtained for informational complexity in this study using local field potentials (LFPs), and several other nonlinear measures such as fractal dimension and other entropy methods (Ma et al., 2018), but not to other measures such as power in different

343 bands and connectivity methods. This finding allows us to interpret that LZ may index 344 behaviourally defined wakefulness, or arousability by stimuli (Bonnet et al., 1978). Although 345 ketamine is used as an anesthetic and creates unconsciousness in high doses and hence can be 346 framed in terms of consciousness as wakefulness and arousal, the effects at lower doses require a multidimensional framework, able to accommodate neurological symptoms (dizziness, slurred speech), mood modulations, and psychedelic experiences. In principle, if ketamine had the classic profile of a sedative, responsiveness would monotonically decrease (Brown et al., 2011) and a similar profile would be expected for molecular and neural measures. However, ketamine has an interesting profile as it belongs to a group of hypnotics that show hallucinatory capacities and an hormetic or U-shaped curve (Calabrese \& Baldwin, 2001) in EEG and blood flow (Cavazzuti et al., 1987; Tsuda et al., 2007). The hormesis of the dose response allows for the comparison of not only conscious level in the sense of wakefulness but in terms of contents of consciousness in low ketamine and REM sleep. From humans we know that the likelihood of increased richness in 356 mental content during the sleep-wake cycle occurs during REM (Windt \& Noreika, 2011) after a 357 decrease in NREM (U-shaped); and we know that the richness of mental content, including hallucinations, peaks early with ketamine before decreasing into sedation and anesthesia (Powers et al., 2015) (an inverted U-shaped curve). In both cases the higher levels of content agree with the higher (or recovering) levels of informational complexity as measured by LZ (Abásolo et al., 2015; Mateos et al., 2018; Schartner et al., 2015; Schartner, Carhart-Harris, et al., 2017; Schartner,

362 Pigorini, et al., 2017). In this study, we compare the consistency of the complexity in the cortex in 363 sleep and the diversity in the ketamine challenge as two putatively very different mechanisms of 364 reaching a higher level of content in consciousness. 
Recent findings by Mediano et al. (2020) provide strong quantitative evidence on how environmental conditions have a substantial influence on neural dynamics during a psychedelic experience in humans. This work showed how brain entropy is modulated by stimulus manipulation during a psychedelic experience by studying participants under the effects of LSD or placebo, either with gross state changes (eyes closed vs. open) or different stimulus (no stimulus vs. music vs. video). Results showed that while brain entropy increased with LSD in all the experimental conditions, it exhibited largest changes when subjects have their eyes closed, whereas the entropy enhancing effects of LSD were less marked when participants opened their eyes or perceived external stimuli — such as music or video (Mediano et al., 2020). In the present work, we studied the modulation of auditory stimulation on brain complexity in basal conditions and under increasing doses of ketamine in 3 cats using LFP recordings with the hypothesis of observing a higher level of complexity under stimulation. However, only a slight increase in LZ was evidenced during stimulation in dorsolateral prefrontal and auditory cortices, whereas a complete lack of or very weak effect were found in the other cortices studied (Figure 4). This weak effect may be explained by the low relevance of the stimulus, as it failed to catch the attention of the animals, compared to extremely salient or meaningful stimuli such as music or video. Further evidence that stimulation studies should exploit more complex stimuli also comes from a recent study were TMS pulses also failed to increase complexity in low doses of ketamine in humans (Farnes et al., 2020). New experiments using more appropriate stimuli in terms of relevance and salience are needed to better address this hypothesis and further the experimental understanding neural dynamics of information theory, complexity and entropy as the system is modulated pharmacologically.

Our sleep results are consistent with previous results in humans (Andrillon et al., 2016; M. Schartner et al., 2017), as well as in rats (Abasolo et al., 2015). However, a closer read shows some differences: Andrillon et al. (2016) reported a small but reliable decrease in LZ during REM sleep

392 compared to the waking state, possibly due to participants engaged in a task during the waking 393 state, whereas the participants in the Schartner et al. study were simply at rest with eyes closed and 394 not engaged or externally driven by task or stimuli. In our study the animals were also at rest but 395 with eyes open and showed a decrease in LZ during LS and further decrease in SWS, which was similar for all cortices (Figure 2A) in line with previous findings (Andrillon et al., 2016; M. 
Schartner et al., 2017). However, a greater variability was evident for REM sleep state where in

398 some cortices LZ was equal in level of complexity to wakefulness whereas in others it was similar

399 to LS or to SWS (Figure 2B). The complexity pattern among sleep stages observed in the cortex

400 was also evidenced the lateral geniculate nucleus (Figure 2A), lending clear convergent evidence

401 to the common effects of informational complexity in the brain beyond the cortex for the sleep

402 wake cycle.

404 In summary, our data demonstrate that there is a dose-dependent ketamine effect on neural 405 complexity. An increase in complexity compared to baseline was found for some cortices 406 (prefrontal, motor, auditory and visual) only in the lowest doses, while the higher dose frequently 407 showed the lowest informational complexity. However, a decrease in complexity was also seen in 408 somatosensory and posterior parietal cortex in the low doses. The heterogeneity of the ketamine 409 effects between cats and cortices contrasts with the homogeneity of the changes in complexity seen 410 for different stages of sleep, further highlighting the differences between natural and 411 pharmacologically induced changes in consciousness. The individual and cortical variability in the 412 neural complexity dynamics revealed by ketamine highlights the intricacy of the brain when 413 perturbed by dissociatives and psychedelics, pushing for a multidimensional framework beyond 414 simple arousal and alertness parameters to characterize the change in the states of consciousness 415 from a neuropharmacological perspective.

\section{Methods}

421 Five adult cats were used in this study; all of whom were also utilized in a previous report (Castro422 Zaballa et al., 2019). The animals were obtained from and determined to be in good health by the 423 Institutional Animal Care Facility of the Faculty of Medicine (University of the Republic, 424 Uruguay). All experimental procedures were conducted in accordance with the Guide for the Care 425 and Use of Laboratory Animals (8th edition, National Academy Press, Washington DC, 2011) and 426 were approved by Institutional and National Animal Care Commissions of the University of the 427 Republic in Uruguay (Protocol No 070153000089-17). Adequate measures were taken to minimize 
428 pain, discomfort or stress to the animals. In addition, all efforts were made to use the minimum

429 number of animals necessary to produce reliable scientific data.

\section{Surgical procedure}

433 Following general anesthesia, the head was positioned in a stereotaxic frame and the skull was 434 exposed. Stainless steel screw electrodes (1.4 mm diameter) were placed on the surface (above the 435 dura matter) of different cortical areas including prefrontal, primary motor, primary somatosensory 436 and posterior parietal cortices. Note that because the animals were not prepared specifically for 437 this work, we did not analyse the same cortices in all of them. The electrodes were connected to a 438 Winchester plug, which together with two plastic tubes were bonded to the skull with acrylic 439 cement in order to maintain the animals' head in fixed position without pain or pressure. After 440 recovery from surgical procedures, they were adapted to the recording environment for a period 441 of at least 2 weeks.

\section{Data acquisition and preprocessing}

445 Experimental sessions of $4 \mathrm{~h}$ were conducted between 11 a.m. and 3 p.m. in a temperature446 controlled environment $\left(21-23{ }^{\circ} \mathrm{C}\right)$. During these sessions (as well as during the adaptation 447 sessions), the animals' head was held in a stereotaxic position by four steel bars that were placed 448 into the chronically implanted plastic tubes, while the body rested in a sleeping bag (semi-restricted 449 condition).

451 The ECoG activity was recorded with a monopolar (referential) configuration, utilizing a common 452 reference electrode located in the left frontal sinus. The electromyogram (EMG) of the nuchal 453 muscles, which was recorded by means of an acutely placed bipolar electrode, was also monitored. 454 The electrocardiogram (ECG), by electrodes acutely placed on the skin over the pre-cordial region, 455 and respiratory activity by means of a micro-effort piezo crystal infant sensor were also recorded. 456 Each cat was recorded daily for $\sim 30$ days in order to obtain complete basal and treatment data 457 sets. 
Bioelectric signals were amplified $(\times 1,000)$, filtered $(0.1-500 \mathrm{~Hz})$, sampled $\left(1,024 \mathrm{~Hz}, 2^{16}\right.$ bits $)$ and stored in a PC using the Spike 2 software (Cambridge Electronic Design).

Data were obtained after ketamine administration as well as during spontaneously occurring quiet W, LS, NREM sleep and REM sleep (Fig. 1). Five, 10, and $15 \mathrm{mg} / \mathrm{kg}$ i.m. of ketamine (Ketonal ${ }^{\circledR}$, Richmond Veterinaria S.A.) were administered to five animals. These three doses were administered four times in each animal in different experimental sessions in a counterbalanced order (each animal received 12 doses of ketamine). The recovery time between consecutive ketamine experiments was $72 \mathrm{~h}$. Ketamine $(50 \mathrm{mg} / \mathrm{ml})$ was diluted in benzethonium chloride, hydrochloric acid, and water (solution for veterinary use). Basal recordings (without injections) were used as control. Sound stimuli during $300 \mathrm{~s}$ were applied $10 \mathrm{~min}$ after ketamine injection in three cats. These sound stimuli had the same characteristics as those used to induce active W (Castro et al., 2013).

Sound stimuli was applied for a period of $300 \mathrm{~s}$; in drug-free recordings and following ketamine administration. In drug-free condition, the stimuli were introduced 30 min after the beginning of the recording sessions. The sound consisted of continuous stimulus of 60-100 dB SPL with a variable frequency of presentation $(1-500 \mathrm{~Hz}$, modified at random) in order to avoid habituation (Castro et al., 2013; Torterolo et al., 2003). Sound stimuli during $300 \mathrm{~s}$ were also applied $10 \mathrm{~min}$ after ketamine injection in three cats.

For preprocessing, sleep stages were scored off-line by visual inspection of 5-s epochs in Spike2 software, where the ECoG and electromyogram (EMG) were displayed simultaneously. In order to analyze LZs during sleep, a total of 300 artifact-free seconds data were selected from each 483 behavioral state. Additionally, to study LZs during the Ketamine effect 300 s duration segments, with and without stimulation, were selected before and after ketamine administration. preprocessing. The Matlab toolbox eeglab was used to filter the data (0.5-200 Hz band-pass). Each epoch was visually inspected, and those with gross artifacts (e.g. movements) were removed from the analysis. 


\section{Lempel-Ziv complexity}

493 In this study we used Lempel-Ziv (LZ) complexity to compute the complexity of measured neural 494 signals (Lempel \& Ziv, 1976). In particular, we used the LZ78 algorithm (Ziv \& Lempel, 1978), 495 which corresponds to the standard word-dictionary implementation: given a binary string, the 496 algorithm scans it sequentially looking for distinct structures or "patterns." The more diverse the 497 binary string, the more patterns are included in the dictionary (a sequence containing only zeros or only ones would lead to the minimal number of patterns being obtained). The total number of these patterns is a measure of signal diversity.

To compute LZ from our experimental data, the recording of each channel was split into segments of 5120 samples $(5 \mathrm{~s}$ sampled at $1024 \mathrm{~Hz})$. Then, to generate a discrete sequence from a real-valued signal $\mathrm{X}$ of length $T, \mathrm{X}$ is detrended and binarized with a threshold of 0 , and the resulting binary sequence is fed to the LZ78 algorithm. Finally, the resulting dictionary length $L$ is normalized as

$$
C=\frac{\log _{2} L}{T} L
$$

to yield a measure of complexity $C$.

Statistics

512 One way ANOVA, with Tukey post-hoc test were used to compare LZ between sleep stages per 513 cortex per animal (Fig. 2B) where Cohen's d was used to address the size of the effect. 514 Additionally, a multilevel approach as well as Bayesian Informational Criterion (BIC) were used 515 to find the most likely explanatory model within the hierarchical model in the group statistical 516 analysis comparing linear, quadratic and cubic models. For sleep study, the state of sleep was used 517 as fixed effect and the cat identity as random effect. The same type of approach was used to study 518 the ketamine effect among different cortices under control and stimulus conditions. In this case the 519 dose and stimulus (if present) were used as fixed effects; and cat identity and session as random 
effects. The interaction between stimuli and ketamine dose was also included in the model when studying the modulation by stimulus. All models were estimated via restricted maximum likelihood, using the open-source packages lme4 v.1.1-21 (Bates et al., 2015) and lmerTest v.3.11 (Kuznetsova et al., 2017) on R v.3.6.1.

\section{Acknowledgements}

This study was supported by the "Programa de Desarrollo de Ciencias Básicas, PEDECIBA" and the "Comisión Sectorial de Investigación Científica" (CSIC) I + D-2020-393 grant from Uruguay. PAM and DB are funded by the Wellcome Trust (grant no. 210920/Z/18/Z).

\section{References}

Abásolo, D., Simons, S., Morgado da Silva, R., Tononi, G., \& Vyazovskiy, V. V. (2015). Lempel-Ziv complexity of cortical activity during sleep and waking in rats. Journal of Neurophysiology, 113(7), 2742-2752. https://doi.org/10.1152/jn.00575.2014

Amargós-Bosch, M., López-Gil, X., Artigas, F., \& Adell, A. (2006). Clozapine and olanzapine, but not haloperidol, suppress serotonin efflux in the medial prefrontal cortex elicited by phencyclidine and ketamine. International Journal of Neuropsychopharmacology, 9(5), 565-573. https://doi.org/10.1017/S1461145705005900 of responsiveness to the environment in human sleep. Journal of Neuroscience, 36(24), 6583-6596. https://doi.org/10.1523/JNEUROSCI.0902-16.2016

Bates, D., Mächler, M., Bolker, B. M., \& Walker, S. C. (2015). Fitting linear mixed-effects models using lme4. Journal of Statistical Software, 67(1). https://doi.org/10.18637/jss.v067.i01

Bekinschtein, T., Cologan, V., Dahmen, B., \& Golombek, D. (2009). You are only coming 
through in waves: wakefulness variability and assessment in patients with impaired consciousness. Progress in Brain Research, 177(C), 171-189. https://doi.org/10.1016/S0079-6123(09)17712-9

Blain-Moraes, S., Lee, U., Ku, S., Noh, G., \& Mashour, G. A. (2014). BlainMoraes2014.pdf. Front Syst Neurosci., 8, 114. https://doi.org/10.3389/fnsys.2014.00114

Bonnet, M., Hohnson, L., \& Webb, W. (1978). The reliability of arousal threshold during sleep. Psychophysiology, 15(5), 412-416.

Brown, E. N., Purdon, P. L., \& Van Dort, C. J. (2011). General Anesthesia and Altered States of Arousal: A Systems Neuroscience Analysis. Ann Rev Neurosci., 34, 601-628. https://doi.org/10.1146/annurev-neuro-060909-153200

Calabrese, E. J., \& Baldwin, L. A. (2001). Hormesis: A generalizable and unifying hypothesis. Critical Reviews in Toxicology, 31(4-5), 353-424. https://doi.org/10.1080/20014091111730

Carhart-Harris, R. L., Kaelen, M., Bolstridge, M., Williams, T. M., Williams, L. T., Underwood,

Carhart-Harris, Robin L. (2018). The entropic brain - revisited. Neuropharmacology, 142, 167178. https://doi.org/10.1016/j.neuropharm.2018.03.010 diethylamide (LSD). Psychological Medicine, 46(7), 1379-1390.

Carhart-Harris, Robin L., Leech, R., Hellyer, P. J., Shanahan, M., Feilding, A., Tagliazucchi, E., Chialvo, D. R., \& Nutt, D. (2014). The entropic brain: A theory of conscious states informed by neuroimaging research with psychedelic drugs. Frontiers in Human Neuroscience, 8(1 FEB), 1-22. https://doi.org/10.3389/fnhum.2014.00020

Casali, A. G., Gosseries, O., Rosanova, M., Boly, M., Sarasso, S., Casali, K. R., Casarotto, S., Bruno, M. A., Laureys, S., Tononi, G., \& Massimini, M. (2013). A theoretically based index of consciousness independent of sensory processing and behavior. Science Translational Medicine, 5(198). https://doi.org/10.1126/scitranslmed.3006294

Castro-Zaballa, S., Cavelli, M. L., Gonzalez, J., Nardi, A. E., Machado, S., Scorza, C., \& Torterolo, P. (2019). EEG $40 \mathrm{~Hz}$ coherence decreases in REM sleep and ketamine model of psychosis. Frontiers in Psychiatry, 10(JAN), 1-14. https://doi.org/10.3389/fpsyt.2018.00766

Castro, S., Falconi, A., Chase, M. H., \& Torterolo, P. (2013). Coherent neocortical 40-Hz 
oscillations are not present during REM sleep. European Journal of Neuroscience, 37(8), 1330-1339. https://doi.org/10.1111/ejn.12143

Cavazzuti, M., Porro, C. A., Biral, G. P., Benassi, C., \& Barbieri, G. C. (1987). Ketamine effects on local cerebral blood flow and metabolism in the rat. 7, 806-811. https://doi.org/DOI: 10.1038/jcbfm.1987.138

Chen, X., Shu, S., \& Bayliss, D. A. (2009). HCN1 channel subunits are a molecular substrate for hypnotic actions of ketamine. Journal of Neuroscience, 29(3), 600-609. https://doi.org/10.1523/JNEUROSCI.3481-08.2009

Conti, F., Minelli, A., Molnar, M., \& Brecha, N. C. (1994). Cellular localization and laminar

Farnes, N., Bjørn, E. J., Nilsen, A. S., Romundstad, L. G., \& Storm, J. F. (2020). Increased signal. PLoS One, 23. https://doi.org/10.1371/journal.pone.0242056

Ferenets, R., Lipping, T., Anier, A., Jäntti, V., Melto, S., \& Hovilehto, S. (2006). Comparison of entropy and complexity measures for the assessment of depth of sedation. IEEE Transactions on Biomedical Engineering, 53(6), 1067-1077. https://doi.org/10.1109/TBME.2006.873543

Ferenets, R., Vanluchene, A., Lipping, T., \& Heyse, B. (2007). Behavior of Entropy / Complexity Measures of the Electroencephalogram during Propofol-induced Sedation. Anesthesiology, 106(4), 696-706.

Ferrer-Allado, T., Brechner, V. L., Dymond, A., Cozen, H., \& Crandall, P. (1973). Ketamineinduced Electroconculsice Phenomena in the Human Limbic and Thalamic Regions. Anesthesiology, 38, 333.

González, J., Cavelli, M., Castro-Zaballa, S., Mondino, A., Tort, A. B. L., Rubido, N., Carrera, Rubido, N. (2019). Decreased electrocortical temporal complexity distinguishes sleep from wakefulness. Scientific Reports, 9(1), 1-9. https://doi.org/10.1038/s41598-019-54788-6 
Effects on Prefrontal Cortex Interneurons and Pyramidal Neurons. J Neurosci., 27, 1149611500. https://doi.org/10.1523/JNEUROSCI.2213-07.2007

Huntley, G. W., Vickers, J. C., Janssen, W., Brose, N., Heinemann, S. F., \& Morrison, J. H. (1994). Distribution and synaptic localization of immunocytochemically identified NMDA receptor subunit proteins in sensory-motor and visual cortices of monkey and human. Journal of Neuroscience, 14(6), 3603-3619. https://doi.org/10.1523/jneurosci.14-0603603.1994

Ishizawa, Y., Ahmed, O. J., Patel, S. R., Gale, J. T., Sierra-Mercado, D., Brown, E. N., \& Eskandar, E. N. (2016). Dynamics of propofol-induced loss of consciousness across primate neocortex. Journal of Neuroscience, 36(29), 7718-7726. https://doi.org/10.1523/JNEUROSCI.4577-15.2016

Kuznetsova, A., Brockhoff, P. B., \& Christensen, R. H. B. (2017). lmerTest Package: Tests in Linear Mixed Effects Models . Journal of Statistical Software, 82(13). https://doi.org/10.18637/jss.v082.i13

Langsjo, J. W., Maksimow, A., Salmi, E., Kaisti, K., Aalto, S., Oikonen, V., Hinkka, S., Aantaa,

Lee, U., Ku, S., Noh, G., Baek, S., Choi, B., \& Mashour, G. A. (2013). Disruption of FrontalParietal Communication by Ketamine, Propofol, and Sevoflurane. Anesthesiology, 118, 1264-1275. https://doi.org/10.1097/ALN.0b013e31829103f5

Lempel, A., \& Ziv, J. (1976). On the Complexity of Finite Sequences. IEEE Transactions on Information Theory, 22(1), 75-81. https://doi.org/10.1109/TIT.1976.1055501 induced by ketamine. NeuroImage, 196(March), 32-40. 
644 López-Gil, X., Jiménez-Sánchez, L., Campa, L., Castro, E., Frago, C., \& Adell, A. (2019). Role of Serotonin and Noradrenaline in the Rapid Antidepressant Action of Ketamine. ACS Chemical Neuroscience, 10(7), 3318-3326. https://doi.org/10.1021/acschemneuro.9b00288

López-Gil, X., Jiménez-Sánchez, L., Romón, T., Campa, L., Artigas, F., \& Adell, A. (2012). Importance of inter-hemispheric prefrontal connection in the effects of non-competitive NMDA receptor antagonists. International Journal of Neuropsychopharmacology, 15(7), 945-956. https://doi.org/10.1017/S1461145711001064

Lorrain, D. S., Schaffhauser, H., Campbell, U. C., Baccei, C. S., Correa, L. D., Rowe, B., Rodriguez, D. E., Anderson, J. J., Varney, M. A., Pinkerton, A. B., Vernier, J. M., \& Bristow, L. J. (2003). Group II mGlu receptor activation suppresses norepinephrine release in the ventral hippocampus and locomotor responses to acute ketamine challenge.

Mediano, P. A. M., Rosas, F. E., Timmermann, C., Roseman, L., Nutt, D. J., Feilding, A., Neuropsychopharmacology, 28(9), 1622-1632. https://doi.org/10.1038/sj.npp.1300238

Ma, Y., Shi, W., Peng, C. K., \& Yang, A. C. (2018). Nonlinear dynamical analysis of sleep electroencephalography using fractal and entropy approaches. Sleep Medicine Reviews, 37, 85-93. https://doi.org/10.1016/j.smrv.2017.01.003

Mateos, D. M., Guevara Erra, R., Wennberg, R., \& Perez Velazquez, J. L. (2018). Measures of entropy and complexity in altered states of consciousness. Cognitive Neurodynamics, 12(1), 73-84. https://doi.org/10.1007/s11571-017-9459-8 Kaelen, M., Kringelbach, M. L., Barrett, A. B., Seth, A. K., Muthukumaraswamy, S., Bor, D., \& Carhart-Harris, R. L. (2020). Effects of external stimulation on psychedelic state neurodynamics. BioRxiv, 2020.11.01.356071. https://doi.org/10.1101/2020.11.01.356071

Moghaddam, B., Adams, B., Verma, A., \& Daly, D. (1997). Activation of Glutamatergic Neurotransmission by Ketamine: A Novel Step in the Pathway from NMDA Receptor Blockade to Dopaminergic and Cognitive Disruptions Associated with the Prefrontal Cortex. The Journal of Neuroscience, 17(8), 2921-2927. https://doi.org/10.1523/JNEUROSCI.17-08-02921.1997

Pal, D., Li, D., Dean, J. G., Brito, M. A., Liu, T., Fryzel, A. M., Hudetz, A. G., \& Mashour, G. A. (2020). Level of consciousness is dissociable from electroencephalographic measures of cortical connectivity, slow oscillations, and complexity. Journal of Neuroscience, 40(3), 605-618. https://doi.org/10.1523/JNEUROSCI.1910-19.2019 
675 Pascovich, C., Castro, S., Velasquez, N., Bor, D., Canales-Johnson, A., Torterolo, P., \& Bekinschtein, T. A. (2019). Complexity of cortical activity under subanesthetic doses of ketamine and during sleep. https://osf.io/dvpyr

Powers, A. R., Gancsos, M. G., Finn, E. S., Morgan, P. T., \& Corlett, P. R. (2015). KetamineInduced Hallucinations (pp. 376-385). https://doi.org/10.1159/000438675

Sarasso, S., Boly, M., Napolitani, M., Gosseries, O., Charland-Verville, V., Casarotto, S., Rosanova, M., Casali, A. G., Brichant, J. F., Boveroux, P., Rex, S., Tononi, G., Laureys, S.,

Schartner, M. M., Carhart-Harris, R. L., Barrett, A. B., Seth, A. K., \& Muthukumaraswamy, S. \& Massimini, M. (2015). Consciousness and complexity during unresponsiveness induced D. (2017). Increased spontaneous MEG signal diversity for psychoactive doses of ketamine, LSD and psilocybin. Scientific Reports, 7(March), 1-12. https://doi.org/10.1038/srep46421

Schartner, M. M., Pigorini, A., Gibbs, S. A., Arnulfo, G., Sarasso, S., Barnett, L., Nobili, L.,

Schartner, M., Seth, A., Noirhomme, Q., Boly, M., Bruno, M. A., Laureys, S., \& Barrett, A.

Schwartz, M. S., Viden, S., \& Scott, D. F. (1974). Effects of ketamine on the electroencephalograph. Anaesthesia, 29(2), 135-140. https://doi.org/10.1111/j.13652044.1974.tb00611.x

Seamans, J. (2008). Losing inhibition with ketamine. Nature Chemical Biology, 4, 91-93.

Tagliazucchi, E., Carhart-Harris, R., Leech, R., Nutt, D., \& Chialvo, D. R. (2014). Enhanced repertoire of brain dynamical states during the psychedelic experience. Human Brain Mapping, 35(11), 5442-5456. https://doi.org/10.1002/hbm.22562

Timmermann, C., Roseman, L., Schartner, M., Milliere, R., Williams, L. T. J., Erritzoe, D., Muthukumaraswamy, S., Ashton, M., Bendrioua, A., Kaur, O., Turton, S., Nour, M. M., Day, C. M., Leech, R., Nutt, D. J., \& Carhart-Harris, R. L. (2019). Neural correlates of the 
DMT experience assessed with multivariate EEG. Scientific Reports, 9(1), 1-13. https://doi.org/10.1038/s41598-019-51974-4

Tononi, G., Boly, M., Massimini, M., \& Koch, C. (2016). Integrated information theory: From consciousness to its physical substrate. Nature Reviews Neuroscience, 17(7), 450-461. https://doi.org/10.1038/nrn.2016.44

Tononi, G., \& Edelman, G. M. (1998). Consciousness and complexity. Science, 282(5395), 1846-1851. https://doi.org/10.1126/science.282.5395.1846

Torterolo, P., Yamuy, J., Sampogna, S., Morales, F. R., \& Chase, M. H. (2003). Hypocretinergic Neurons are Primarily involved in Activation of the Somatomotor System. Sleep, 26(1), 2528. https://doi.org/10.1093/sleep/26.1.25

Tsuda, N., Hayashi, K., Hagihira, S., \& Sawa, T. (2007). Ketamine, an NMDA-antagonist,

Windt, J. M., \& Noreika, V. (2011). How to integrate dreaming into a general theory of consciousness-A critical review of existing positions and suggestions for future research. Consciousness and Cognition, 20(4), 1091-1107. https://doi.org/10.1016/j.concog.2010.09.010 R., Albuquerque, E. X., Thomas, C. J., Zarate, C. A., \& Gould, T. D. (2018). Ketamine and

Zhou, C., Douglas, J. E., Kumar, N. N., Shu, S., Bayliss, D. A., \& Chen, X. (2013). Forebrain HCN1 channels contribute to hypnotic actions of ketamine. Anesthesiology, 118(4), 785795. https://doi.org/10.1097/ALN.0b013e318287b7c8 